expression of the four types of resultant transgenic mice ES cells were observed. The expressions of the targeting genes were further evaluated through RT-PCR and Western-blot techniques. MTT method was employed to investigate the cellular proliferation and flow cytometry was used to analyse the rate of apoptosis.

Results After co-transfection of 293 FT cells with four types of plasmids, the fluorescence microscopic observation showed that all four types of lentivirus particles (hHCN4-hTbx3-eGFP, hHCN4-eGFP, hTbx3-eGFP and eGFP) were successfully constructed which was then confirmed by both RT-PCR and Western-blot. Virus titre of above were $\quad 4.7 \times 107 \mathrm{TU} / \mathrm{ml}, \quad 5.2 \times 107 \mathrm{TU} / \mathrm{ml}, \quad 4.5 \times 107 \mathrm{TU} / \mathrm{ml}$ and $6.65 \times 107 \mathrm{TU} / \mathrm{ml}$, respectively. After being transfected by the 4 types of lentivirus, the cells in both hHCN4-eGFP and eGFP group had better cellular morphology and a relatively higher fluorescence rate than that in both hTbx3-eGFP and hHCN4-hTbx3-eGFP groups. Comparing to normal ES cell group, there was no significant difference of the cellular proliferation in both hHCN4-eGFP $(p=0.25)$ and eGFP ( $p=0.08$ ) groups by MTT method, while the rates of cell growth in both Tbx3-eGFP and hHCN4-hTbx3-eGFP groups were dramatically decreased $(p<0.01)$. The rates of cell apoptosis in the four groups (hHCN4-hTbx3-eGFP, hHCN4-eGFP, hTbx3-eGFP and eGFP) were $74.53 \%, 16.29 \%, 67.64 \%$ and $13.06 \%$ respectively.

Conclusions The lentiviral vectors that simultaneously carrying with HCN4, Tbx3 and eGFP gene were successfully constructed, and also the corresponding highly infectious lentivirus particles were packed, which were effective in the transfection of ES cells. Tbx3 appeared not to promote the proliferation of ES cell, in contrast, to facilitate the apoptosis of ES cell.

\section{GW23-e1212 IMPACT OF HUMAN HCN4 AND TBX3 GENE CO- TRANSFECTED LENTIVIRUS ON BIOLOGICAL ACTIVITY OF EMBRYONIC STEM CELL (ES)}

doi:10.1136/heartjnl-2012-302920b.43

Li Ping, Dong Hao, Wang Li Jun, Li Ping. Cardiovascular Department, The Second Affiliated Hospital to NanChang University

Objectives T-box3 (Tbx3) is a family member of the T-box (Tbx) gene, which is a family of transcription factors involved in the regulation of developmental processes. The previous study showed that Tbx3 induced a high-level expression of sinoatrial node (SAN)-specific gene at the heart-transplant region. Hyperpolarisation-activated cyclic nucleotide-gated channel 4 (HCN4 gene), the most abundant subtype of $\mathrm{HCN}$ gene in sinoatrial node, played an essential and specific role in the formation of If-channels in the sinoatrial node cells. This study aimed to construct the lentivirus particles that simultaneously carrying with human HCN4 gene, Tbx3 gene and enhanced green fluorescence protein (eGFP) gene and then investigate the impact of coexpression of HCN4 and Tbx3 on the biological activity of mice embryonic stem cell. Results may provide a foundation for further study of rebuilding sinoatrial node cell function through the co-expression of HCN4 and Tbx3.

Methods By using the liposome approach, four types of targetinggene-bound subject plasmids (hHCN4-hTbx3-eGFP, hHCN4-eGFP, hTbx3-eGFP and eGFP) and a helper plasmid were packaged respectively. The 293 FT cells were co-transfected with these four types of plasmids respectively, and the virus titre of each type of co-transfected cells was then calculated. Afterwards, the transduction of mice ES cells by the four types of resultant lentiviral plasmid were performed respectively. The morphologies and the degrees of fluorescence 